Revue musicale OICRM

\title{
Rester en retrait de l'espace médiatique. Le cas Schreker
}

\section{Aurore Flamion}

Volume 7, numéro 1, 2020

Écrits de compositeurs et espaces médiatiques

URI : https://id.erudit.org/iderudit/1069472ar

DOI : https://doi.org/10.7202/1069472ar

Aller au sommaire du numéro

Éditeur(s)

Observatoire interdisciplinaire de création et recherche en musique (OICRM)

ISSN

2368-7061 (numérique)

Découvrir la revue

Citer cet article

Flamion, A. (2020). Rester en retrait de l'espace médiatique. Le cas Schreker. Revue musicale OICRM, 7(1), 78-95. https://doi.org/10.7202/1069472ar

\section{Résumé de l'article}

Principalement connu pour ses opéras, le compositeur Franz Schreker (1878-1934) connaît une trajectoire des plus étonnantes : si ses premiers opéras sont salués par la presse, il subit rapidement le feu de critiques extrêmement diverses. Outre les arguments ad personam - il est issu d'une famille juive -, la presse s'empare de réseaux sémantiques qui étayent cet antisémitisme : ceux de la décadence et de la dégénérescence. Particulièrement féconds dans la presse germanique des années 1918-1921, ces termes ne semblent pas susciter de réponse de Schreker, qui préfère publier ses essais esthétiques dans les Musikblätter des Anbruch, jeune journal qu'il se sait acquis. Le compositeur se tient loin des polémiques : politique ou esthétique, aucune ne suscite de véritable réponse. Définissant sa démarche compositionnelle dans un nombre restreint d'articles publiés dans les Musikblätter des Anbruch, Schreker semble en effet évacuer toute référence aux termes concentrant la polémique : décadence, dégénérescence, impressionnisme et expressionnisme. 


\title{
Rester en retrait de l'espace médiatique. Le cas Schreker
}

\author{
Aurore Flamion
}

\section{Résumé}

Principalement connu pour ses opéras, le compositeur Franz Schreker (18781934) connaît une trajectoire des plus étonnantes : si ses premiers opéras sont salués par la presse, il subit rapidement le feu de critiques extrêmement diverses. Outre les arguments ad personam - il est issu d'une famille juive -, la presse s'empare de réseaux sémantiques qui étayent cet antisémitisme : ceux de la décadence et de la dégénérescence. Particulièrement féconds dans la presse germanique des années 1918-1921, ces termes ne semblent pas susciter de réponse de Schreker, qui préfère publier ses essais esthétiques dans les Musikblätter des Anbruch, jeune journal qu'il se sait acquis. Le compositeur se tient loin des polémiques : politique ou esthétique, aucune ne suscite de véritable réponse. Définissant sa démarche compositionnelle dans un nombre restreint d'articles publiés dans les Musikblätter des Anbruch, Schreker semble en effet évacuer toute référence aux termes concentrant la polémique : décadence, dégénérescence, impressionnisme et expressionnisme.

Mots clés : esthétique ; opéra ; presse ; retrait médiatique ; Franz Schreker.

\begin{abstract}
Mainly known for his operas, the composer Franz Schreker (1878-1934) had a rather uncommon path: though his first operas got hailed by critics, he quickly came under fire for various reasons. In addition to the ad personam attacks- he came from a Jewish family - the press responded with semantic networks that supported this anti-Semitism: the leitmotiv of decadence and degeneration. Quite popular in the Germanic press of 1918-1921, these terms did not seem to trigger a response from Schreker, who preferred to publish his aesthetic essays in the Musikblätter des Anbruch, a new Austrian paper that supported him. The composer was far from polemics, wether they be political or aesthetic. Instead, he described his compositional approach in a limited number of articles published in the Musikblätter des Anbruch, and he did not seem to make any reference to the controversial terms: decadence, degeneration, impressionism and expressionism.
\end{abstract}

Keywords: aesthetics; opera; press; Franz Scheker; withdrawal form media. 
S'inscrire dans les réseaux médiatiques, y affirmer sa singularité - de critique, ou de compositeur : impossible, à l'ère du journal, de s'y soustraire. A contrario, tout silence - temporaire ou prolongé - intrigue, tant l'on conçoit mal que l'artiste ne puisse, ou ne veuille, s'exprimer au sujet de son art. De prolifiques critiques furent tentés d'élaborer de véritables stratégies en la matière : fatigué par les échanges animés qu'il nourrit pourtant lui-même dans divers journaux français, Hector Berlioz (1861) n'hésite pas à fustiger $1^{\prime}$ " infernale chaîne du feuilleton ${ }^{1}$ ", quand Robert Schumann, après près de dix années au Neue Zeitschrift für Musik, se retire de l'espace médiatique. Quelques décennies plus tard, Maurice Ravel semble plus durablement confronté à ce silence : celui qui déclarait en 1928, dans son Esquisse autobiographique, "ne jamais [avoir] éprouvé le besoin de formuler, soit pour autrui soit pour [lui] - même, les principes de [s]on esthétique " (cité dans Orenstein 1989, p. 47) s'est tenu à une prudente distance de l'espace médiatique, craignant notamment que ses propos soient modifiés.

La question dela presse, principal espace médiatique au début du $\mathrm{xx}^{\mathrm{e}}$ siècle, est contre toute attente au cœur de la réception de l'œuvre de Franz Schreker. Le compositeur ne se risque que rarement à écrire dans la presse ${ }^{2}$; pourtant, elle seule semble rendre compte, aujourd'hui, de l'important succès que rencontrèrent les œuvres - principalement des opéras - d'un des compositeurs les plus joués de l'entre-deux guerres. Fournissant des indications précieuses quant à la réception de ces représentations, la presse rend également compte des vives critiques qu'elles suscitent immanquablement. L'analyse de ces discours s'avère alors cruciale en ce qu'elle permet formuler un certain nombre d'hypothèses expliquant l'oubli dans lequel Schreker et son œuvre ont sombré. Une question, récurrente, surgit dès lors que l'on s'intéresse à la réception médiatique du compositeur : pourquoi s'est-il si peu exprimé publiquement ? Quel sens donner à l'absence de réponse aux critiques souvent acerbes qui paraissent dans la presse et attaquent indistinctement ses opéras ou sa personne?

\section{POURQUOI ÉCRIRE ?}

État des lieux

Analyser la relation ambivalente que nourrit Schreker avec la presse ne peut faire l'économie d'un passage en revue des différentes productions du compositeur en la matière. Nous avons choisi de nous concentrer sur la période 1918-1921, période pendant laquelle il connaît ses plus grands succès : Der ferne Klang (Le son lointain, composé entre 1903 et 1910), Die Gezeichneten (Les stigmatisés, 1911-1918) et Der Schatzgräber (Le chercheur de trésors, 1915-1918), trois opéras qui constituent autant

1 Berlioz écrit à son fils le 14 février 1861 : «Encore à présent, crois-tu que ce soit gai, d'être forcé, contraint, de rester à cette infernale chaîne du feuilleton qui se rattache à tous les intérêts de mon existence? » (Berlioz 1995, p. 200).

2 Un tel silence contraste d'autant plus résolument avec la volubilité de ses contemporains : Arnold Schoenberg, Hans Pfitzner ou encore Walter Niemann ont en effet clairement pris position dans les principaux débats musicaux de leur temps. 
de jalons dans la construction de sa carrière. Les stigmatisés rencontre un immense succès dès sa création, comme en témoignent ses reprises à Munich, Nuremberg, Breslau et Dresde pour la saison 1918-1919, rapidement suivies par Vienne, Kiel, Cologne, Berlin et Stuttgart. Le nom de Schreker est abondamment présent dans la presse des années 1910-1920, tant dans les journaux spécialisés - l'on songe au Zeitschrift für Musik, à la jeune revue Melos, créée en 1920, aux Musikblätter des Anbruch pour les audiences nationales, ou aux quotidiens locaux, à l'instar du journal Die Neue Freie Presse - reflétant l'engouement suscité par ses opéras.

Omniprésent dans l'espace médiatique, Schreker n'y prend cependant que très rarement directement part : seuls cinq articles sont écrits et publiés entre 1918 et $1921^{3}$ et paraissent au premier abord quelque peu décevant. Rien ne semble en effet relier entre elles ces prises de positions, qui convoquent des sujets extrêmement divers : rapport à la création esthétique avec "Meine Musikdramatische Idee " " " Mon idée musicale dramatique »] (1919) et " Betrachtungen ${ }^{5}$ » [ "Réflexions»] (1921a) ; retour réflexif sur ses sources d'inspiration avec « Über die Entestehung meiner Opernbücher ${ }^{6}$ » " « $\mathrm{A}$ propos de la genèse de mes livrets d'opéra »] (1920b) ; vagues réflexions sur le statut du directeur de conservatoire avec "Musikhochschule » " Au sujet du conservatoire [de Berlin] »] (1920a), ou encore autoportrait lapidaire avec « Meine Charakterbild » [« Portrait de mon personnage »] (1921b). Hétérogènes dans les sujets qu'ils traitent, ces articles semblent bien souvent répondre à une commande faite au compositeur, ou s'inspirent très largement de lettres préexistantes, adressées à son ami Paul Bekker (voir Hailey 1994) ; l'inscription du compositeur dans l'espace médiatique paraît loin d'être spontanée. Elle évacue, de façon surprenante, toute intertextualité ; Schreker ne conçoit pas ses articles comme une réponse aux critiques que connaissent ses œuvres, mais comme la possibilité d'évoquer à grands traits les sujets qui l'importent - ou qui sont censés l'importer.

L'absence de références à l'univers bruissant des journaux n'est pas la seule à étonner ; la fonction auctoriale ${ }^{7}$, sur le plan discursif, paraît également complètement désinvestie par Schreker, qui ne se prononce jamais sur la nature de ses œuvres, leurs propriétés ou leurs valeurs ; aucune mention, également, d'hypothétiques prescriptions en matière d'interprétation. Il ne s'aventure pas non plus dans le domaine analytique ; aucune analyse d'œuvre, même partielle.

3 Christopher Hailey (1993) fait état de 25 articles écrits entre 1909 et 1933 fréquemment (re)publiés dans l'Anbruch ; le compositeur s'exprime principalement au sujet de l'opéra, de la crise qu'il connaît et des perspectives qu'elle offre, de son rapport à la composition ou l'enseignement, et de ses œuvres.

$4 \quad$ L'article reprend des éléments évoqués dans des lettres adressées à son ami et critique Paul Bekker. Ce court essai lui avait été demandé par l'almanach de l'Opéra et du Schauspielhaus de Francfort pour la saison 1917-1918.

$5 \quad$ Article auquel nous n'avons pas pu avoir accès, recensé par Christopher Hailey (1993) et qui n'est pas (ou infimement) évoqué par la littérature scientifique portant sur l'œuvre de Schreker.

$6 \quad$ L'article reprend une lettre écrite à Bekker le 18 juin 1918 (Hailey 2014).

7 Nous entendons ici, par «fonction auctoriale ", tout ce qui «fait d'un auteur un auteur » : en l'occurrence, comment Schreker ne semble pas investir spontanément, de lui-même, la place, le discours ou le statut du compositeur en tant qu'auteur, c'est-à-dire en tant qu'instance de régulation de son œuvre, pouvant par là même faire l'objet d'une herméneutique ou d'une mythographie. 


\section{Les fondements d'une esthétique}

Pourquoi Schreker se prête-t-il donc - certes, rarement - à l'exercice ? Si le compositeur ne semble pas animé de la volonté de rendre précisément compte de sa démarche compositionnelle, certains de ses articles lui permettent, malgré un certain degré de généralité, d'affirmer les grands principes de sa personnalité musicale.

Les deux courts essais « Meine Musikdramatische Idee » (1919) et "Über die Entstehung meiner Opernbücher »(1920b), publiés à quelques mois d'écart et reprenant tous deux les discussions que Schreker échange avec son ami Bekker, s'avèrent fondamentaux pour comprendre les quelques éléments donnés par Schreker dans la presse.

Le premier entend poser un certain nombre de jalons permettant de saisir la singularité de ses œuvres. Schreker justifie une démarche compositionnelle fondée sur l'intrication de la musique et du drame : ce dernier s'impose à lui, lui indique les grandes lignes de l'œuvre et peut expliquer l'apparente absence de principe formel cohérent ou rigoureux (" mon idée musicale dramatique ? Je n'en ai pas vraiment. J'écris sans but ${ }^{8} » ;$ Schreker 1919, p. 6). C'est précisément alors l'article « Über die Entstehung meiner Opernbücher " qui permet de donner un contenu aux principes énoncés par Schreker; pour expliciter l'idéal de fusion du mot et du son qui fait l'objet de "Meine Musikdramatische Idee ", il revient ici sur cinq de ses opéras : Der ferne Klang, Das Spielwerk und die Prinzessin ("La princesse et la boîte à musique ", 1908-1911), Die Gezeichneten, Der Schatzgräber et Irrelohe ("Les flammes démentes » 1919-1922), relatant pour chacun d'entre eux des souvenirs, véritables stimulus poétiques extérieurs.

Schreker opte ici également pour des formulations extrêmement vagues, n'allant pas davantage dans l'explicitation de la naissance de l'œuvre et se contentant de motivations psychologiques assez banales : "Le son lointain est né de mes doutes. En moi-j'étais encore un jeune homme, cela mûrissait ${ }^{9}$ "; ou encore : "j' ai moi-même écrit Le son lointain, à partir de ma propre expérience ${ }^{10}$ " (ibid., p. 547-548). Schreker, pourtant, en avait proposé une toute autre lecture quelques années plus tôt, alors passionnément épris de Grete Jonasz, jeune pianiste résidant dans le même immeuble que lui ${ }^{11}$ (Perroux 2001, p. 27).

Les lignes consacrées à Das Spielwerk und die Prinzessin permettent de saisir quant à elles ce qui fonde la spécificité d'une démarche compositionnelle restée abstraite dans 1'article « Meine Musikdramatische Idee »(Schreker 1919). Recourant à des

\footnotetext{
8 "Ich schreibe planlos. Was mir einfällt, ist da. Nur - ich komme von der Musik her». Par défaut, les traductions du présent article sont des traductions libres.

9 "Aus zwiefacher Not heraus entstand der "Ferne Klang". In mir - ich war ein ganz junger Mensch - gärte es. "

10 "Und schrieb den „Fernen Klang”, aus mir selbst heraus, aus meinem eigenen jungen Erleben. »

11 Les lettres qu'il lui écrit en 1907 sont éloquentes ; le compositeur affirme ainsi sans ambages à la jeune femme, dans une lettre du 30 janvier : «Vraiment, Grete, je te rends grâce d'avoir mis tout cela en marche. Je suis si étonné lorsque j'observe mon - pardon : ton œuvre - elle se tient plus grande que nature devant moi ».
} 
descriptions minutieuses, le récit se sert de l'hypotypose pour proposer une progression fondée sur la condensation de trois épisodes : la scène d'un vieux violoniste jouant pour la foule réunie pour lui dans son village natal, que Schreker a lue dans un journal, rencontre le souvenir d'une soirée d'été dans les vignobles de Grinzings, à la périphérie de Vienne, où il croit reconnaître le violoniste. Le traumatisme d'une manifestation violente quelques temps plus tard achève de donner naissance à l'opéra.

Un fil relie cette succession d'épisodes ; Schreker émaille son texte d'expressions mettant en scène l'acte même de composer ("J'ai terminé le travail un autre soir ${ }^{12}$ », «Et moi, écrivant fébrilement ${ }^{13}$ », Schreker 1919, p. 548). Schreker, ce faisant, contribue à forger l'image d'un compositeur tout entier consacré à son art : l'inspiration surgit des évènements de sa vie et intrique fondamentalement idée dramatique et composition musicale. Le portrait que le compositeur dessine de lui-même, dans ces deux articles, demeure singulièrement empreint de romantisme : loin de toute théorisation, Schreker affirme la force créatrice d'une sensibilité s'appropriant des éléments biographiques, prosaïques, pour les traduire immédiatement, presque involontairement, en inspiration musicale.

\section{Schreker pédagogue}

La composition et l'enseignement occupent assurément une place importante dans la vie de Schreker : professeur de composition au conservatoire de Vienne dès 1912, il est nommé directeur de la Hochschule de Berlin en mars 1920. L'article attaché à cette activité, "Musikhochschule » (Schrecker 1920a) énonce un certain nombre de principes pédagogiques, mais demeure extrêmement général (attirer les plus grands musiciens doués d'une véritable appétence pour l'enseignement, favoriser l'épanouissement de ses étudiants, valoriser la singularité de ces derniers ainsi que les échanges avec d'autres établissements), à l'instar des articles consacrés aux principes compositionnels.

On est rapidement en droit de s'interroger : pourquoi Schreker, compositeur, chef d'orchestre, professeur et directeur de la Hochschule de Berlin, n'a-t-il pas davantage écrit ? Il semble assez peu probable que l'auteur de Die Gezeichneten ait manqué d'occasions pour partager son expérience... tout comme paraît peu plausible le refus, par les différents journaux, des écrits qu'il aurait écrits : Schreker bénéficie sans conteste de l'amitié des journaux Melos (créé en 1920) et des Musikblätter des Anbruch (créées en 1919), ayant ouvertement pris parti pour la musique " moderne » à laquelle semble initialement appartenir celle de Schreker.

De ce corpus singulier et devant l'absence de certitudes expliquant le retrait de Schreker, émergent alors plusieurs hypothèses constituant autant de fils nous permettant de tenter de saisir plus nettement les intentions du compositeur. Deux d'entre elles nous semblent particulièrement prégnantes : celle d'un désintérêt et d'une méfiance de la part de Schreker pour l'activité de critique et celle, complé-

12 "Ich vollendete das Werk an einem anderen Abend. »

13 «Et moi, écrivant fébrilement, je me suis soudainement retrouvé coincé dans une foule terrible » ("Und ich - fieberhaft schreibend - sah mich plötzlich eingekeilt in furchtbarem Gedränge »). 
mentaire, d'une incapacité à revenir dans un texte écrit sur ses pratiques en tant que compositeur.

\section{Quelle est L'IMAGe De SCHREKer CRÉÉE PAR LA PRESSE?}

\section{La décadence comme esthétique}

Avant de tenter de cerner plus précisément les raisons de l'éloignement médiatique de Schreker, il convient de se pencher sur productions discursives le prenant précisément pour objet. D'emblée, le lecteur est confronté au biographisme ${ }^{14}$ irriguant ces articles, encore caractéristique des discours tenus sur l'art et qui postule une transitivité pouvant être totale entre les qualités personnelles de l'artiste et celles de son œuvre. Stephan Downes (2010) souligne ainsi à quel point peu d'ouvrages musicologiques s'attachent à déplier les différentes formes d'une décadence spécifiquement musicale, malgré un paradigme omniprésent dans le répertoire du début $\mathrm{xx}^{\mathrm{e}}$ siècle. Cet angle mort, dans le cas Schreker, s'accompagne d'un certain paradoxe : si les paradigmes de la décadence ("Dekadenz ») et de la dégénérescence ("Entartung ») ${ }^{15}$ sont omniprésents dans la critique, ils sont rarement convoqués sous ces noms dans la presse de l'époque.

Le lecteur qui souhaiterait ainsi consulter ces textes afin d'en dégager les principaux griefs motivant la décadence ou la dégénérescence musicales supposées de Schreker serait par ailleurs vite déçu, tant ces essais ne recourent pas - ou très peu - à l'analyse de la partition ou du livret ${ }^{16}$. Ne se référant pas précisément à l'œuvre en tant qu'objet, imprégnés d'une culture de la critique journalistique, ces discours restent en surface de l'œuvre, investissant au contraire des schèmes discursifs n'étant a priori pas circonscrits au domaine musical, mais participant d'une culture commune. L'analyse de ce type de discours met ainsi en évidence une certaine intersectionnalité des paradigmes ou des idéologies irriguant ces textes : idéologies genrées, sexistes, antisémites ${ }^{17}$ ou plus

14 Marcel Proust, dans son Contre Sainte-Beuve (1954, posthume, p. 131) enjoint à distinguer l'artiste en tant qu'homme et en tant qu'instance créatrice, considérant qu'il n'est pas nécessairement de continuité entre eux ; il fustige ce faisant la "méthode Sainte-Beuve », qui « consiste à ne pas séparer l'homme et l'œuvre ».

15 Max Bourget, avec sa "théorie de la décadence " (Essais de psychologie contemporaine, 1881), mais également Richard Wagner ("Éclaircissements sur Le Judaïsme dans la musique», 1850 ; Opéra et drame, 1851) et Friedrich Nietzsche (Nietzsche contre Wagner, Le cas Wagner, Le crépuscule des idoles, 1889) constituent trois figures majeures ayant écrit sur la décadence. Max Nordau (La dégénérescence, 1892) et George Bernard Shaw (The Sanity of Art. An Exposure of the Current Nonsense about Artists being Degenerate, 1908) ont quant à eux évoqué plus spécifiquement le paradigme de la dégénérescence.

16 Si les critiques ne se réfèrent pas précisément aux opéras pour justifier leurs reproches, ceux-ci semblent se focaliser sur la conception du son défendue par Schreker : une texture sonore (le Klang schrekérien, texture timbre-son) fondée sur la fréquente division des pupitres ou le recours fréquent au glockenspiel et au célesta créant une texture irisée aux reflets moirés ; le prélude de Die Gezeichneten en est l'incarnation la plus éloquente.

17 Nous empruntons ici la définition proposée par Pierre-André Taguieff (2015, p. 9) : « Stricto sensu, le mot "antisémitisme" signifie un rejet des Juifs fondé sur des arguments empruntés à une doctrine moderne pseudo-scientifique de la race (qui les catégorise en tant que "Sémites"), à une forme de racialisme (théorie descriptive, évaluative et explicative) ou de racisme (théorie normative et prescriptive, incluant un programme politique)». Les ouvrages d'Amaury du Closel (2005) et d'Élise Petit (2018) rendent particulièrement compte du passage d'un antisémitisme latent à un antisémitisme politique. 
généralement racistes s'emparent d'un vocable commun pour discriminer un même objet. Omniprésente dans la critique de l'époque, la décadence n'est paradoxalement jamais définie, ce qui semble, comme le souligne Richard Gilman (1979) être un de ses traits constitutifs : son « existence est purement négative "; il s'agit " d'un mot choisi pour remplir un espace » (cité dans Downes 2010, p. 30). Il est néanmoins possible d'esquisser une typologie de ses présentations principales - au nombre de six - dans les discours consacrés à Schreker :

a) La peur d'une inintelligibilité du texte : les critiques déplorent le manque de cohérence de l'œuvre. En témoignent les propos de Julius Korngold, l'une des personnalités musicales les plus férocement opposées à Schreker, qui condamne le caractère informe d'une langue ne se tenant plus d'elle-même tant au niveau de la macrostructure que de la microstructure ${ }^{18}$ : il considère que la forme, inintelligible, est fondée sur l'oubli de l'harmonie; celle-ci est « décousue ${ }^{19}$ » (Korngold 1920, p. 4).

b) L'autonomisation d'éléments normalement hiérarchisés : la décadence fonde également l'obscurité du langage auquel elle recourt sur la dé-hiérarchisation des différents paramètres de l'œuvre, dontl'un prédomine exagérément. Albert Noelte, critique musical et compositeur demande ainsi au sujet de Die Gezeichneten : " la concentration de l'expression musicale et dramatique sur le son (au sens primitif $\mathrm{du}$ mot) dispense-t-elle d'une expression linéaire et mélodique conductrice ${ }^{20}$ ? » (Noelte 1919, p. 1).

c) Une artificialité condamnée : Korngold, d'abord séduit par les trouvailles de Schreker («tout ce qui est simplement coloré est magistral») condamne rapidement son usage du timbre, véritable maquillage des sons qui déguise l'inanité des moyens employés et qui le conduit à « se contenter du matériel de base le plus pauvre, largement utilisé21 » (Korngold 1920, p. 4).

d) Une fascination dangereuse : nombreuses sont les critiques s'alarmant d'une fascination délétère exercée sur l'auditeur. Paul Zschorlich s'inquiète ainsi de leur vulnérabilité : la musique de Schreker aurait « quelque chose de stupéfiant, de berçant, [devant lequel on est] sans résistance ${ }^{22} »(Z s c h o r l i c h ~ 1929$, p. 1).

18 Jean de Palacio (2011, p. 7) considère ainsi que la décadence, véritable " triomphe $\mathrm{du}$ fragment »- l'expression est du poète belge Iwan Gilkin -, représente " tout ce qui mettait en cause l'intégrité de l'Espace, du Temps, du Corps, du Livre, du Texte ». L'absence d'une cohérence interne constitue l'un des traits principaux de la décadence telle qu'elle a été initialement théorisée par Paul Bourget (1908, p. 19-26) ("Un style de décadence est celui où l'unité du livre se décompose pour laisser la place à l'indépendance de la page, où la page se décompose pour laisser la place à l'indépendance de la phrase, et la phrase pour laisser la place à l'indépendance du mot »).

19 "Man fühlt ein geflissentliches Abzielen auf das harmonisch Unzusammenghörige. »

20 "Ist die Konzentration des musikalisch-dramatischen Ausdrucks auf den Klang (im Urbegriff des Wortes) unter Verzicht auf fassbaren melodisch linearen Ausdruck [...]? »

21 «Alles bloß Farbige ist meisterlich. Aber auch die ungewöhnlichsten Klänge können nur einmal ungewöhnliche Melodien, ja nicht einmal gewöhnliche ersetzen, weil Melodie oberster seelischer Ausdruck ist und bleibt. [...] [Er mache] von dem Grundsatz der Radikal-Moderne, sich mit dürftigsten Grundstoff zu begnügen, ausgiebig Gebrauch. »

22 «Etwas Betäubendes, Einlullendes, widerstandslos Machendes. » 
e) L'exacerbation d'une sensualité jouissant d'elle-même : des traductions musicales de la décadence, celle d'une sensualité débridée est peut-être la plus fréquemment rencontrée. Karl Holl (1918) lors de la création de l'œuvre use à cet effet de métaphores lyriques : «des mélodies de grande ampleur dont les lignes courbées et audacieuses, souvent mêlées dans un enchevêtrement bouclé, donnent une vie florissante à la couleur orchestrale ${ }^{23}$ " (cité dans Bujara 2015, p. 153) , quand Noelte déplore le fait que «l'oreille boi[ve] goulûment de cette abondante source de couleur, s'enivre - elle finit par devenir sursaturée, s'émousse et cela, malheureusement même avant l'acte final dramatiquement bouleversé, où la fantaisie sonore de Schreker célèbre de véritables orgies ${ }^{24}$ " (Noelte 1919, p. 1).

f) L'effémination du masculin : enfin, le topos d'une stigmatisation fondée sur le genre afin de discréditer ses rivaux est particulièrement illustré dans la presse musicale, notamment par Bruno Schrader, qui déclare en pensant à Schreker et à ses contemporains : "Tous des efféminés, doux, semblables à des mollusques ${ }^{25}$ ! » (Schrader 1922, p. 218). Un critique anonyme du Darmstädter Tageblatt qualifie Schreker de "nature douce et féminine ${ }^{26}$ " à la suite d'une représentation de Die Gezeichneten, considérant que l'impressionnisme de Schreker est " arrêté avec une atmosphère féminine, sensuelle de l'avant-guerre ${ }^{27}$ " (Anonyme 1926, p. 1115).

\section{Un antisémitisme patent}

Schreker, bien sûr, n'est pas le seul compositeur à se confronter à la violence potentielle des espaces médiatiques et la virulence de ses critiques. À ces polémiques esthétiques, se greffent néanmoins des considérations politiques d'une grande agressivité, comme en témoigne un épisode survenu par numéros interposés dans le Zeitschrift für Musik: Bruno Schrader, compositeur et critique conservateur publie le $1^{\text {er }}$ février 1921 un article ouvertement antisémite. Il oppose au "bon Allemand» (" l'Allemand Michel ») l'élément étranger, donc pathogène, que représente Schreker :

L'invasion sémitique post-tchécoslovaque, qui prospère si bien sous le ministère rouge, continuera-t-elle, ou l'Allemand Michel se réveillera-t-il enfin? Le grand héros de la journée, Schreker, est connu pour avoir un nom complètement différent de ce qu'il prétend être : un seul «numéro » du Tell de Rossini, si rudement blasphémé, est plus ingénieux, plus précieux, et plus immortel qu'une douzaine d'opéras comme ses

23 "[Einer] breit auslandenden Melodik, deren kühn geschwungene, oft in krausen Gewirr durcheinander laufende Linien durch die orchestrale Färbung blühendes Leben erhalten. »

24 "Das Ohr trinkt gierig aus diesem üppigen Farbenquell, berauscht sich daran - wird schließlich übersättigt, abgestumpft und das leider schon vor dem dramatisch bewegten Schlussakt, wo Schrekers Klangphantasie wahre Orgien feiert. »

25 «Alles weibisch, weichlich, molluskenhaft!»

26 "[Schreker] ist eine weibliche, weichliche Natur."

27 «Einer femininen, sinnlich-schwülen Atmosphäre der Vorkriegszeit verhaftet bleibt. » 
Stigmatisés. Ceux qui font l'éloge de tels excréments sont stigmatisés pour toujours ${ }^{28}$ (Schrader 1921a, p. 65).

Devant une telle violence, la rédaction du Zeitschrift für Musik se sent obligée de publier un bref entrefilet dans l'édition du 5 mars 1921 :

Message de l'éditeur.

Diverses communications, suscitées par la section Musikbriefe de Berlin, publiée dans le $3^{\mathrm{e}}$ numéro du journal, nous ont incités à consulter Schreker, et notre collègue. M. Bruno Schrader nous a alors dit : "Je n'ai rien à retirer de mes écrits, ni de mes imprimés ». Il ne se déclare ni partial, ni antisémite, ni engagé politiquement, si ce n'est que la constellation politique interfère directement dans les domaines artistiques $^{29}$ (Anonyme 1921, p. 120).

S'ensuit un dernier épisode dans l'édition du 2 mars 1921, Bruno Schrader n'entendant pas ne pas avoir le dernier mot. Rivalisant de mauvaise foi, il convoque les mêmes arguments antisémites :

Correction. Le compositeur Franz Schreker, à Berlin, citant l'article 11 de la loi sur la presse, annonce que ses parents s'appelaient Schreker et qu'il était baptisé catholique. Il était d'abord hongrois, puis autrichien et est maintenant un ressortissant prussien. Mais cela va bien au-delà de la portée de la correction, qui est décrite avec précision au paragraphe $3 \mathrm{du}$ paragraphe ci- dessus. Le fait que Schreker soit un nom de scène pour Tscharras - l'écriture originale en langue hongroise ou slave ne m'est pas familière - est étayé par trois professeurs de musique célèbres et très respectés, dont l'un travaille dans la Hochschule même Schreker. C'est donc que cela doit être vrai ! Peut-être une erreur? Mais tant de bruit pour une omelette, de surcroît dans la ville cosmopolite de Berlin ${ }^{30}$ ? (Schrader 1921b, p. 166)

28 "Soll nun die semitisch tschecho-slowakische Afterkunstinvasion, die unter dem roten Ministerium so üppig gedeiht, so weitergehen oder wird der deutsche Michel endlich aufwachen? Der großeTagesheld Schreker hat bekanntlich von Haus aus einen ganz andern Namen. Das mag er sich immerhin gesagt sein lassen: eine einzige ,Nummer' von Rossinis neulich so unverständig verlästertem Tell ist genialer, wertvoller und ewiger als ein Dutzend Opern von der Sorte seiner, Gezeichneten'. Diejenigen, welche derlei Kot preisen, sind gezeichnet für immerdar. »

29 "Mitteil ung der Redaktion.

Verschiedene Zuschriften wegen des im Hefte Nr. 3 erschienenen Musikbriefes aus Berlin veranlaßten uns in der Angelegenheit Schreker mit unserem Mitarbeiter Rücksprache zu nehmen. Herr Bruno Schrader teilte uns daraufhin Folgendes mit: ,Ich habe von meinem Geschriebenen bezw. Gedruckten nichts zurückzunehmen'. Ererklärtsich weder für antisemitisch voreingenommen noch politisch anders engagiert, als die politische Konstellation unmittelbar in die künstlerischen Vethältnisse eingreift. »

30 "Berichtigung. Der Komponist Franz Schreker in Berlin teilt unter Berufung auf 11 des Pressgesetzes mit, dass schon sene Eltern Schreker hiessen und er römisch-katholisch getauft ist. Er war zuerst ungarischer, dann österreichischer und ist jetzt preussischer Staatsangehöriger. Doch das überschreitet breits die Grenzen der Berichtigung, die in Absatz 3 des erwähnten Paragraphen, auf den ich mich nun meinerseits versteife, genau umschrieben sind. Dass Schreker ein sog. Künstlername für Tscharras - die ungarische oder slawische Originalschreibart ist mir nicht geläufig-sei, geht von drei berühmten, hochangesehen Musikprofessoren aus, deren einer an Schrekers eigener Hochschule wirkt. Es musste also durchaus glaubwürdig erscheinen. Vielleicht eine Verwechslung? Aber tant de bruit pour une omelette und noch dazu in der Weltstadt Berlin?" 
L'épisode est particulièrement éclairant en ce qu'il met conjointement en lumière les attaques antisémites auxquelles Schreker était coutumier et l'absence d'intervention directe de ce dernier : sa défense est assurée par l'entrefilet correctif du comité de rédaction du Neue Zeitschrift für Musik. Là encore, il faut se résoudre à ces questions sans réponses : Schreker a-t-il tenté de se défendre lui-même et de publier un texte conséquent dans une revue alors ouvertement conservatrice (Alfred Heuss critique et fervent contempteur de la "musique moderne " en est le rédacteur en chef) ? Préfère-t-il se tenir à l'écart des vaines polémiques, espérant que sa relative absence dans la presse, en laissant la machine médiatique tourner à vide, finisse par lasser ses opposants?

\section{DÉSINTÉRÊT ET MÉFIANCE DE SCHREKER POUR LE CHAMP DE BATAILlE MÉDIATIQUE}

En l'absence de prises de positions claires de Schreker, ou de réponse aux critiques esthétiques et polémiques qui lui sont faites, sa correspondance avec son ami Paul Bekker s'est révélée un outil précieux pour tenter de cerner le rapport qu'il entretenait avec la presse.

\section{La critique : une activité méprisée}

Si les articles de Schreker publiés entre 1918 et 1921 semblent témoigner d'une indifférence envers les critiques qui lui sont faites - quelles qu'elles soient -, les lettres qu'il envoie à Bekker montrent qu'il a une excellente mémoire en la matière. Elles font état d'une polarisation extrême entre deux critiques : Bekker lui-même, dont la compréhension de Schreker semble la plus totale, et Julius Korngold, père du compositeur homonyme et critique autrichien de renom. Si ce dernier a contribué à la reconnaissance de Mahler, il est sans appel pour Schreker, dont il dénigre fréquemment les œuvres dans Die Neue Freie Presse, journal viennois dont il est un des rédacteurs musicaux principaux partir de 1902. Les lettres de Schreker témoignent d'une rapide crispation sur la figure de Julius Korngold : il est l'un des seuls critiques nommément évoqués dans une correspondance s'étalant sur plusieurs années (voir Hailey 1994).

Confronté, dans les journaux, à la violence de l'espace médiatique, Schreker se tient, jusqu'à " Mein Charakterbild » à une distance prudente. Il fustige néanmoins le 18 mars 1913 " la misère, la méchanceté, la stupidité et l'envie illimitées » de M. Korngold, qui « ne peut [lui] pardonner le succès du Son lointain » ${ }^{31}$ (ibid., p. 33). Même lorsque le succès semble unanime, à l'instar de la création du Ferne Klang, le nom du critique dissone dans l'allégresse générale : Schreker rapporte ainsi dans une lettre à Bekker, le 9 juillet 1918 (ibid., p. 55) que " pour la première à l'Akademietheater de Vienne, le succès est retentissant, avec des sifflements soutenus (presse enthousiaste jusqu'à M. K[orngold] même, mais assez perplexe) ${ }^{32}$ ».

31 "Dem Wust von Gemeinheit und Gehässigkeit, die grenzlose Dummheit und Neid (Herr Korngold kann mir den Erfolg des ,fernen Klang" nicht verzeihen) in Wien gegen mich aufgeboten hat. »

32 "Bei der Uraufflührung] in Wien im Akademietheater ein starker Erfolg. (Presse bis auf Hr. K[orngold] glänzend aber ziemlich ratlos). " 
Si les attaques de Korngold sont loin de laisser Schreker indifférent, elles s'étendent rapidement, de façon indifférenciée, à l'ensemble des critiques. Dès 1913, Vienne tout entière lui semble hostile, ce dont témoigne une lettre du 18 mars : "Quand je compare votre brillant article au bredouillement désemparé des différents "critiques" ici, j’ai honte de devoir vivre dans cette ville ${ }^{33}$ » (Hailey 1994, p. 33). Schreker déclare à Bekker, plus loin : "Un critique, pardonnez-moi de vous appliquer aussi pour une fois ce mot discrédité, qui exerce son métier en personne sérieuse et sensible, ne peut offenser sérieusement un auteur qui n'est pas un charlatan, même s'il le nie ${ }^{34}$ " (ibid., p. 37).

Le mépris pour Korngold comprend en réalité une misanthropie plus générale, qui, si elle stigmatise une figure en particulier, est loin de se circonscrire au critique et ne tient pas le reste du public en très haute estime ; dans une lettre du 29 mai 1918 le public est qualifié de «foule stupide ${ }^{35}$ » (ibid., p. 33). Le jugement du 9 juillet 1918 est également sans appel : "Soit ces gens ne veulent pas entendre soit ils ne l'écoutent pas soit je suis fou. Mais voyez-vous : nous vivons dans une période si hypocrite $[\ldots]^{36}$ » (ibid., p. 55).

\section{L'incapacité à revenir sur sa pratique de compositeur}

Contrairement à Arnold Schoenberg, collègue et ami de longue date, dont le Traité d'harmonie paraît dès 1911, Schreker ne publie aucun essai théorique : les discussions en matière de composition semblent circonscrites aux salles dans lesquelles il enseigne. Comment analyser, dès lors, ce silence? Faut-il considérer que le compositeur n'a pas eu l'opportunité d'éditer de manuscrit ? Qu'il lui était techniquement impossible de formuler son enseignement sous forme d'essai théorique? Ou enfin, que l'exercice ne l'intéressait tout simplement pas ? Là encore, il nous faut user de sentiers détournés pour étayer ces hypothèses ; les témoignages de quelques-uns de ses élèves insistent fréquemment sur l'absence de dogmatisme du professeur, dont la pédagogie semble principalement fondée sur leur accompagnement vers l'autonomie. Ainsi en est-il d'Alois Hába, qui considère en 1971 que l'enseignement de Schreker possédait «l'ambition de trouver un chemin partant d'une conception traditionnelle et scolaire pour aller vers une indépendance artistique avec une harmonie et un contrepoint fortement altérés » (Perroux 2001, p. 56)... ou de Ernst Krenek, qui affirme que son ancien professeur « avait tout sauf une nature d'érudit, de sorte que son jugement reposait totalement sur l'instinct plutôt que sur l'analyse critique » (ibid., p. 57).

33 "Wenn ich Ihr glänzendes Feuilleton mit dem hilflosen Gestammel diverser „Kritiker” hier vergleiche, so schäme ich mich in dieser Stadt leben zu müssen. "

34 "Ein Kritiker, verzeihen Sie daß ich das in Verruf gekommene Wort auch einmal auf Sie anwende, der seinen Beruf als ernster und fühlender Mensch ausübt, vermag einen Autor, der nicht ein Charlatan ist, auch wenn er verneint, nicht ernsthaft zu kränken. »

35 «Der blöden Masse, genannt Publikum. »

36 "Entweder wollen diese Menschen nicht hören, oder sie hörens nicht oder ich bin verrückt. Aber sehen Sie: Wir leben in einer derart verlogenen Zeit [...]. » 
Schreker semble peiner, ce faisant, à s'approprier le « lien devenu essentiel entre le musicien et l'écrit - support symbolique, s'il en est, de la pensée et de l'intellectualité » (Duchesneau, Dufour et Benoit-Otis 2013, p. 8), nourrissant involontairement les discours remettant en question sa légitimité en tant que compositeur et pédagogue.

Apparemment incapable de revenir sur son enseignement, Schreker ne change pas de stratégie au sujet de ses propres œuvres : il ne publie pas davantage d'article explicitant la singularité de sa production musicale. Ce mutisme repose néanmoins sur certaine incapacité revendiquée : il se dénie toute réflexivité en la matière. Schreker s'inscrit ainsi dans le mythe romantique d'une activité compositionnelle intime, fondée sur une intériorité créatrice ne supportant pas d'être médiatisée : l'œuvre constitue alors ce " point fixe ", cette « origine sans communication avec l'extérieur », au fondement du " fantasme de l'œuvre en soi, dans sa double acception d'œuvre autarcique et d'œuvre au fond de la conscience créatrice » (Maingueneau 2004, p. 34).

Les propos de Schreker, dans une lettre adressée à Bekker le 9 juillet 1918 sont à cet égard éloquents : "Je suis moi-même toujours dans le plus grand embarras quand on me demande quelque chose comme un "programme" d'analyse; cela ne donne généralement rien de très spirituel ${ }^{37}$ " (Hailey 1994, p. 54). Il ajoute, dans la même lettre :

Je suis d'une nature combative dans mes œuvres et, si besoin est, en tant que musicien ou metteur en scène ou même - en tant que directeur musical. Mais une chose ne m'est pas donnée : celle de «s'enfoncer» dans ses propres œuvres. Expliquer m'est déjà pénible, écrire une torture. [...] J'ai travaillé sur la préface du «Spielwerk » plus longtemps que sur l'ensemble du livret ${ }^{38}$ (ibid.).

Cette certitude de ne pas être compris du public peut susciter les stratégies les plus fantasques : Schreker évoque le 18 juin 19181'article «Mon idée musicale dramatique » qui lui a été demandé par le théâtre de Francfort: "Bien sûr, je ne voulais pas être impoli, et je me suis donné bien du mal, à contrecœur, pour mettre en forme quelque chose qui, étant superficiel et creux, ne peut donner aucune image de ce que je voudrais vraiment ${ }^{39}$ » (ibid., p. 47).

Les idées esthétiques défendues par l'article, pour importantes qu'elles soient dans l'esthétique du compositeur, ne demeurent pas moins formulées de manière très générale, comme nous l'avons vu précédemment. Impossible, semble-t-il, de déterminer si les trois questions structurant le texte, brillant ainsi par leur didactisme (« [quelle est mon] idée musicale et dramatique? », " qu'est-ce que je cherche ? et

37 "Ich selbst bin stets in der größten Verlegenheit wenn man von mir so etwas wie ein „Programm” eine Analyse verlangt und es kommt zumeist nichts sehr Geistvolles heraus. "

38 "Aber: Ich bin eine Kampfnatur in meinen Werken und wenn es sein muß, durch energische Tat als Musiker oder Regisseur oder überhaupt - als sich durchsetzender Mensch. Doch eines ist mir gar nicht gegeben. Das ,,Sichversenken" in die eigenen Werke - Erklären ist mir schon peinlich, darüber schreiben eine Qual. »

39 "Ich wollte natürlich nicht unhöflich sein und habe da mit Müh und Not, sehr ungern, etwas zusammengeschrieben, was, hohl und oberflächlich gar kein Bild geben kann von dem was ich eigentlich möchte. " 
" qu'est-ce que je cherche en fin de compte dans mon travail ? "40, Schreker 1919, p. 6-7) ont été imposées à Schreker... ou si ce dernier n'avait d'autre ambition, en soulignant leur relative inanité, que de signifier le refus, ou l'impossibilité d'aller plus avant dans la théorisation de son esthétique.

Une théorisation au second degré?

Schreker n'écrit pas, ou peu, au sujet de son œuvre ; pourtant, son importante correspondance avec Bekker en est parsemée de références : précisions concernant le sens de certains passages, dans la partition ou le livret, remarques quant aux interprètes choisis pour monter les différents opéras, réflexions amères ou rassurées concernant les critiques parues dans la presse... Sckreker trouve en Bekker un ami et son plus grand défenseur. Le critique occupe alors une place singulière, qui n'est pas sans faire penser à celle de Walter Nouvel envers Igor Stravinski, le premier transcrivant les propos du second dans un geste de "corédaction ». À mi-chemin entre la mythographie et la théorisation de l'esthétique schrekérienne, Schreker semble, avec Bekker, adopter « un dédoublement, une posture que l'on peut qualifier d'écriture sans écriture, ou d'écriture au second degré » (Dufour 2013, p. 22). L'un des critiques les plus influents $\mathrm{du} \mathrm{xx}^{\mathrm{e}}$ siècle multiplie en effet les essais qui lui sont consacrés ${ }^{41}$ : parmi ceux-ci, le fondamental ouvrage Studie zur Kritik der modernen Oper (1918). Tout juste contemporain de l'article " Meine musikdramatische Idee ", il établit clairement une filiation entre Schreker et Wagner, installant par là-même le cadre de l'herméneutique schrekérienne, ce que rappelle Carl Dahlhaus dans son article consacré à la dramaturgie du Ferne Klang :

Paul Bekker went further than anyone else as an apologist for Schreker. He praised him as the only modernist composer who, in a similar way to Wagner, though without being stylistically dependent on him, conceived dramas "out of the spirit of music," instead of providing music for literary texts, dramatizing ideas or supplying musical illustration for a piece of cheap theatrical sensationalism (Dahlhaus [1978]1987, p. 193).

\section{La déconstruction d'une figure construite par les médias : un moment schrekerien carnavalesque}

Écrire peu fréquemment des textes caractérisés par l'absence de propos réellement saillants : Schreker semble s'être tenu à cette ligne de conduite jusqu'à la publication du détonnant autoportrait qu'il publie en 1921 dans les Musikblätter des Anbruch. Alain Perroux (2001, p. 96) dans la monographie qu'il lui consacre, y voit une réponse directe aux médisances proférées dans les journaux, qui vont croissant, et dont Schreker semble également prendre progressivement conscience ; le $1^{\mathrm{er}}$ avril 1921, Schreker rompt avec la stratégie médiatique employée jusque-là en choisissant un journal dont les affinités esthétiques étaient identiques aux siennes (voir

40 «Meine musikdramatische Idee?[...] Was ich erstrebe? [...] Was ich letzten Endes für mein Schaffen erstrebe? »

41 Le site Internet de la fondation Schreker dénombre de 32 essais - articles et livres confondus consacrés en partie ou totalement à Schreker. 
Schreker 1921b, p. 128) : paraissant entre 1919 et 1937, le journal viennois publié par Universal-Edition s'attachait à donner aux défenseurs de la " musique moderne » un réel espace médiatique pour exprimer leurs idées. Theodor W. Adorno, Béla Bartók, Alban Berg, Paul Bekker, Arnold Schoenberg ou encore Hans Heinz Stuckenschmidt écrivent régulièrement au sujet de questions contemporaines ${ }^{42}$ : outre les numéros consacrés à certains compositeurs (Mahler, Busoni, Bartok, Schoenberg, Schreker...), le journal s'intéresse également à quelques sujets polémiques : la question du jazz (Stefan 1925), celle du " problème de la Nouvelle Musique » (Stefan 1926) ou encore celle de la politisation de l'univers musical (Stefan 1931).

\section{Portrait de mon personnage}

Je suis impressionniste, expressionniste, internationaliste, futuriste, vériste musical ; juif et ayant fait son chemin grâce au pouvoir du judaïsme, chrétien et "fabriqué " par une clique catholique placée sous le patronage d'une princesse viennoise archi-catholique. Je suis artiste du son, esprit chimérique du son, magicien $\mathrm{du}$ son, esthète du son, et n'ai pas le moindre vernis de mélodie (excepté quelques figures, pour ainsi dire, de style, qu'on appelle depuis peu des "petites mélodies »). Je suis un mélodiste du sang le plus pur, mais - bien qu'étant un musicien pur-sang - je suis pourtant un harmoniste anémique et pervers ! Je suis (malheureusement) érotomane et agis de façon nuisible sur le public allemand (l'érotisme est selon les apparences mon invention la plus authentiquement personnelle, malgré Les noces de Figaro, Don Giovanni, Carmen, Tannhäuser, Tristan, La Walkyrie, Salomé, Elektra, Le chevalier à la rose et autres). Mais je suis aussi idéaliste (Dieu merci !) et symboliste, je me situe à l'aile la plus radicale des modernes (Schoenberg, Debussy), je ne suis pourtant pas tout à fait à gauche, je suis inoffensif dans ma musique, j'utilise des triples accords, même encore l'accord de septième diminuée totalement " trivial », je m'appuie sur Verdi, Puccini, Halévy et Meyerbeer ; je suis absolument singulier et spécule sur les instincts des masses, je suis un compositeur dramatique pour le cinéma ; je suis un homme " tirant ses forces de la nostalgie et de la morbidité "; j'écris de manière exclusivement homophone, ma musique est pure et propre, subtile, alambiquée, une mer pleine de sons agréables, un amoncellement terrible de cacophonies ; je suis, contrairement à d'autres, un héros de la réclame de la plus mauvaise espèce, "plein de vin doux ", " un document grandiose du déclin de notre culture ", un fou, une tête calculatrice, un chef d'orchestre misérable mais aussi une personnalité de la direction d'orchestre, je suis un technicien brillant, je suis capable de ne pas diriger une fois mes œuvres (et je les dirige constamment); je suis en tout cas un "phénomène ", je suis en outre un mauvais poète mais un bon musicien [...], ma musique jaillit de ma poésie, ma poésie de ma musique, je suis aux antipodes de Pfitzner, le seul successeur de Wagner, un concurrent de Strauss et de Puccini, je flatte le public, j'écris seulement pour fâcher tout le

42 On compte également parmi les contributeurs les plus réguliers de la revue Paul Amadeus Pisk, ancien élève de Schoenberg et de Schreker, qui la dirigera d'octobre 1920 à avril 1922 et cofondateur de l'International Society for Contemporary Music (ISCM), et Paul Stefan, ancien élève de Schoenberg qui assurera la direction du journal à partir de 1922. 
monde et ai eu récemment l'idée d'émigrer vraiment au Pérou. Ce que - pour l'amour du ciel - je ne suis pas ? Je n'ai pas (encore) déraillé, je n'ai pas la folie des grandeurs, je ne suis pas aigri, je ne suis pas un ascète, un gâcheur ou un dilettante et je n'ai jamais encore écrit de critique ${ }^{43}$.

Auparavant caractérisé par un effacement extrême, Schreker atteint en 1921 un point de non-retour qui signe une explosion cynique et sarcastique de tous les cadres référentiels lui ayant été apposés. Reprenant méthodiquement toutes les expressions dont il a été qualifié au moyen d'anaphores à l'efficacité redoutable, il ridiculise ses adversaires sans avoir à opposer de réelle réponse : discréditant tout jugement, il ne répond pas à ses contempteurs pour autant, et ne donne aucun élément infirmant, ou confirmant ces expressions ; l'ironie suffit à suspendre tout jugement. Schreker impose ainsi un cadre rhétorique confortable dans lequel ni locuteur, ni locutaire n'ont de réelle épaisseur : usant du discours indirect libre, il ne singularise aucun critique en particulier, mais les discrédite tous. Cette réfutation systématique des arguments d'autrui entretenue par cette stratégie rhétorique lui permet ainsi de n'avoir à opposer aucun contre-argument. S'il prend paradoxalement la forme d'une définition identitaire très forte, son manifeste en sape en réalité tous les fondements, tant le compositeur affirme l'impossible substantification de son œuvre et de sa personne.

Lassé - pour ne pas dire irrité - par la profusion de discours lui assignant une identité qu'il refuse, Schreker est ici particulièrement habile ; si, comme le considère Paul Valéry, " écrire c'est entrer en scène - il ne faut pas que l'auteur proclame qu'il n'est pas comédien. On n'y échappe pas » (Valéry 1931, p. 1218), Schreker refuse de prendre part à cet espace médiatique profondément étranger à sa nature. Ne stigmatisant aucun critique en particulier, ne réfutant aucun des termes qui lui a été apposé, il se contente, au moyen d'antiphrases, d'en souligner les cohabitations absurdes : esthétique idiosyncratique du son, caractère sulfureux de sa musique ou de ses livrets, concurrence des catégories esthétiques, compétences et singularité du compositeur et du chef d'orchestre - rien n'est épargné. Le seul texte signant une réelle présence de son autorité renvoie, ici encore, à une chaise vide : plus encore que les articles commandés par autrui, ou peu investis par Schreker, « Meine Charakterbild » témoigne de la lassitude éprouvée face à l'absurde coexistence des critiques les plus diverses, et de son refus de se positionner esthétiquement. Il est, enfin, l'occasion de revenir sur son silence en matière critique : Schreker y consacre les derniers mots de son article, ayant pleinement conscience de déroger à ce que l'on attend de lui ; que penser, dès lors, de son énigmatique « encore » [ "noch»] : s'agit-il d'une ultime provocation, le compositeur n'ayant aucune intention de se prêter à l'exercice?

Bref épisode burlesque où le compositeur s'approprie sarcastiquement les différents masques supposés être les siens, l'article ne constitue finalement qu'une forme de variation autour de son silence médiatique, et en entérine l'absolue nécessité.

43 Voir Dictéco pour l'article original (Schreker 1921b). Traduction de « Meine Charakterbild » présentée dans un programme de concert au Théâtre des Champs-Élysées, en 2009, reprise dans le livret accompagnant les représentations de Die Gezeichneten à l'Opéra de Lyon, en 2015, révisée par Alexandre Iagodkine. 


\section{CONCLUSION}

S'inscrire dans les réseaux médiatiques de son temps : Schreker semble n'y avoir consenti qu'avec mauvaise grâce. Principalement issus de commandes ( Meine Musikdramatische Idee »), demandés par les circonstances ("Musikhochschule ») ou issus de lettres échangées avec Bekker, les articles publiés dans les années les plus intenses de sa carrière se résument à quelques principes esthétiques et pédagogiques, sans que Schreker ne prenne directement exemple sur ses propres œuvres. La réflexivité induite par l'exercice, rendant inconfortable la théorisation de son esthétique, semble également pouvoir expliquer l'absence d'essai consistant en la matière. L'absence de réaction aux critiques parues dans la presse, quant à elle, paraît fondée sur le peu d'estime que Schreker portait à cette activité.

L'espace médiatique créé par les journaux bruissant de voix multiples, paradoxalement, constitue un huis clos pour Schreker. Outre la violence journalistique réelle à laquelle est confrontée Schreker dès ses plus grands succès, se dessine une autre violence, symbolique et ontologique : celle d'une insupportable définition identitaire, dont les contours, une fois esquissés, ne peuvent subir aucune modification. Répondre aux critiques, accepter d'entrer dans cette relation identitaire : autant d'actions résolument opposées à une démarche compositionnelle, singulièrement romantique, ne supportant pas d'être médiatisée.

\section{BIBLIOGRAPHIE}

Anonyme (1921) «Musikbriefe. Mitteilung der Redaktion », Zeitschrift für Musik, vol. 88, n 5 (5 mars), p. 120.

Anonyme (1926) " Hessisches Landestheater. ,Die Gezeichneten'. Oper von Franz Schreker », Darmstädter Tagblatt, n 279 (29 novembre), p. 1115.

Berlioz, Hector (1995), Correspondance générale, dir. Pierre Citron, "Vol. VI. 1859-1963 », Paris, Flammarion.

Bekker, Paul ([1918]1983), Franz Schreker. Studie zur Kritik der modernen Oper, Aachen, Rimbaud Presse.

Bourget, Paul ([1881]1908), Essais de psychologie contemporaine, Paris, Plon-Nourrit.

Bujara, Karsten (2015), Klang - Farbe - Geschlecht - Sexualität. Diskursive Metaphorik nationaler Identität I Alterität in der Rezeptionsgeschichte der musikalischen Moderne am Beispiel des Komponisten Franz Schreker, thèse de doctorat, Université Humboldt, Berlin.

Dahlhaus, Carl ([1978] 1987), "Schreker and Modernism. On the Dramaturgy of "Der ferne Klang" », dans Dahlhaus, Schoenberg and the New Music. Essays by Carl Dahlhaus, traduit de l'allemand par Derrick Puffett et Alfred Clayton, Cambridge, Cambridge University Press, p. 192-199.

Downes, Stephen (2010), Music and Decadence in European Modernism. The Case of Central and Eastern Europe, New York, Cambridge University Press.

Duchesneau, Michel, Valérie Dufour, et Marie-Hélène Benoit-Otis (2013), Écrits de compositeurs. Une autorité en questions, Paris, Vrin.

Dufour, Valérie (2013) «Le dédoublement du compositeur. Autorité et rhétorique de Stravinski au miroir de ses écrits », dans Duchesneau, Dufour et Benoit-Otis 2013, p. 17-25.

Du Closel, Amaury (2005), Les voix étouffées du III Reich. Entartete Musik, Paris, Actes Sud. 
Gilman, Richard (1979), Decadence. The Strange Life of an Epithet, New York, Farrar Straus \& Giroux.

Hailey, Christopher (1993), Franz Schreker, 1878-1934. A Cultural Biography, Cambridge, Cambridge University Press.

Hailey, Christopher (dir.) (1994), Briefwechsel. Mit sämtlichen Kritiken Bekkers über Schreker, Aachen, Éditions Rimbaud.

Holl, Karl (1918), «Franz Schreker. Die Gezeichneten: Uraufführung im Frankfurter Opernhaus am 25. April 1918 ", Allgemeine Musik-Zeitung, vol. 45, nº 18, p. 209-221.

Korngold, Julius (1920), « Operntheater. Die Gezeichneten: Oper von Franz Schreker », Neue Freie Press Wien, vol. 56, no 19937 (28 février), p. 1-4.

Maingeneau, Dominique (2004), Le discours littéraire. Paratopie et scène d'énonciation, Paris, Armand Colin.

Nietzsche, Friedrich ([1889a]1991), Nietzsche contre Wagner et Le cas Wagner, édité par Mazzino Montinari et Giorgio Colli, traduit de l'allemand par Jean-Claude Hémery, Paris, Gallimard.

Nietzsche, Friedrich ([1889b]1988), Le crépuscule des idoles, traduit de l'allemand par JeanClaude Hémery, Paris, Gallimard.

Noelte, Albert (1919), «Die Gezeichneten. Oper in drei Aufzügen von Franz Schreker: erste Aufführung am Nationaltheater in München ", München-Augsburger Abendzeitung, vol. 8, $\mathrm{n}^{\circ} 83$ (17 février), p. 1.

Nordau, Max ([1892]1909), Dégénérescence, traduit de l'allemand par Dietrich Auguste, Paris, F. Alcan.

Orenstein, Arbie (dir.) (1989), Maurice Ravel. Lettres, écrits, entretiens, Paris, Flammarion.

Palacio, Jean de (2011), La décadence. Le mot et la chose, Paris, Les Belles Lettres.

Perroux, Alain (2001), Franz Schreker. À la recherche du son lointain, Troinex, Éditions Papillon.

Petit, Élise (2018), Musique et politique en Allemagne du IIt Reich à l'aube de la guerre froide, Paris, Presses de l'Université Paris-Sorbonne.

Proust, Marcel (1954), Contre Sainte-Beuve, édition posthume, Paris, Gallimard.

Schoenberg, Arnold (1911), Harmonielehre, Vienne, Universal-Edition.

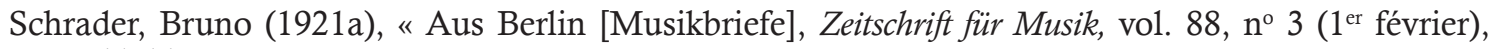
p. 64-66.

Schrader, Bruno (1921b), «Aus Berlin [Musikbriefe] », Zeitschrift für Musik, vol. 88, nº 6 (2 mars) p. 165166.

Schrader, Bruno (1922), "Aus Berlin [Musikbriefe] ", Zeitschrift für Musik, vol. 89, n 9-10 (avril), p. 218-219.

Schreker, Franz (1919) "Meine Musikdramatische Idee ", Musikblätter des Anbruch, vol. 1, n 1 (novembre), p. 6-7.

Schreker, Franz (1920a), "Musikhochschule », Musikblätter des Anbruch, vol. 2, n 14, (septembre), p. $490-493$.

Schreker, Franz (1920b), «Über die Entstehung meiner Opernbücher », Musikblätter des Anbruch, vol. 2, $\mathrm{n}^{\circ} 16$, (octobre), p. 547-549.

Schreker, Franz (1921a), «Betrachtungen », Blätter der Staatsoper, vol. 1, n 3, p. 2-3.

Schreker, Franz (1921b), "Mein Charakterbild», Musikblätter des Anbruch, vol. 3, n 7 (avril), p. 128.

Shaw, George Bernard (1908), The Sanity of Art. An Exposure of the Current Nonsense About Artists Being Degenerate, Londres, The New Age Press.

Stefan, Paul (dir.) (1925), Musikblätter des Anbruch, vol. 7, $\mathrm{n}^{\circ} 4$ (avril). 
Stefan, Paul (dir.) (1926), Musikblätter des Anbruch, vol. 8, $\mathrm{n}^{\circ} 6$ (juin-juillet).

Stefan, Paul (dir.) (1931), Musikblätter des Anbruch, vol. 13, n 1 (janvier).

Taguieff, Pierre-André (2015), L'antisémitisme, Paris, Presses Universitaires de France.

Valéry, Paul (1931), Cahiers, tome 2, Paris, Gallimard.

Wagner, Richard (1850), « Éclaircissements sur Le Judaïsme dans la musique », Neue Zeitschrift für Musik, vol. $33, \mathrm{n}^{\circ} 19$ (3 septembre), p. 101-107.

Wagner, Richard ([1851]1928), " Opéra et drame », traduit de l'allemand par J-G Prod'homme, Euvres complètes de Richard Wagner, Paris, Delegrave.

Weissmann, Adolf (1921), "Die Gezeichneten. Franz Schreker in der Staatsoper ", B.Z. am Mittag, vol. 44, $\mathrm{n}^{\circ} 6$ (6 janvier), [s.p.].

Zschorlich, Paul (1929), "Die Gezeichneten von Schreker. Städtische Oper », Deutsche Zeitung, vol. 34, $\mathrm{n}^{\circ} 283$ (3 décembre), p. 1. 